\title{
BOUNDARY VALUE PROBLEMS FOR ELLIPTIC PSEUDO-DIFFERENTIAL OPERATORS
}

\author{
KAZUAKI TAIRA
}

(Communicated by Barbara Lee Keyfitz)

\begin{abstract}
The purpose of this paper is to study boundary value problems for elliptic pseudo-differential operators which originate from the problem of existence of Markov processes in probability theory. We prove existence and uniqueness theorems for these boundary value problems in the framework of Sobolev spaces. Our approach permits us to interpret sufficient conditions for the unique solvability of boundary value problems in terms of Markovian motion.
\end{abstract}

\section{INTRODUCTION AND RESULTS}

Let $\Omega$ be a bounded domain of Euclidean space $\mathbf{R}^{n}$, with $C^{\infty}$ boundary $\partial \Omega$; its closure $\bar{\Omega}=\Omega \cup \partial \Omega$ is an $n$-dimensional, compact $C^{\infty}$ manifold with boundary.

Let $W$ be a second-order, elliptic pseudo-differential operator with real coefficients such that

$$
\begin{aligned}
W u(x)= & P u(x)+S_{r} u(x) \\
:= & \left(\sum_{i, j=1}^{n} a^{i j}(x) \frac{\partial^{2} u}{\partial x_{i} \partial x_{j}}(x)+\sum_{i=1}^{n} b^{i}(x) \frac{\partial u}{\partial x_{i}}(x)+c(x) u(x)\right) \\
& +\int_{\Omega} s(x, y)\left[u(y)-\sigma(x, y)\left(u(x)+\sum_{j=1}^{n}\left(y^{j}-x^{j}\right) \frac{\partial u}{\partial x_{j}}(x)\right)\right] d y .
\end{aligned}
$$

Here:

(1) $a^{i j} \in C^{\infty}\left(\mathbf{R}^{n}\right), a^{i j}=a^{j i}$ and there exists a constant $a_{0}>0$ such that

$$
\sum_{i, j=1}^{n} a^{i j}(x) \xi_{i} \xi_{j} \geq a_{0}|\xi|^{2}, \quad x \in \mathbf{R}^{n}, \xi \in \mathbf{R}^{n}
$$

(2) $b^{i} \in C^{\infty}\left(\mathbf{R}^{n}\right)$.

(3) $c \in C^{\infty}\left(\mathbf{R}^{n}\right)$ and $c \leq 0$ in $\Omega$.

Received by the editors January 10, 1994.

1991 Mathematics Subject Classification. Primary 35S15, 35J25; Secondary 60J60, 60J50.

Key words and phrases. boundary value problems, pseudo-differential operators. 
(4) The integral kernel $s(x, y)$ is the distribution kernel of a properly supported, pseudo-differential operator $S \in L_{1,0}^{2-\kappa}\left(\mathbf{R}^{n}\right), \kappa>0$, which has the transmission property with respect to the boundary $\partial \Omega$ (cf. [Bo], [Ho], [RS]), and $s(x, y) \geq 0$ off the diagonal $\left\{(x, x) ; x \in \mathbf{R}^{n}\right\}$ in $\mathbf{R}^{n} \times \mathbf{R}^{n}$. The measure $d y$ is the Lebesgue measure on $\mathbf{R}^{n}$.

(5) The function $\sigma(x, y)$ is a $C^{\infty}$ function on $\bar{\Omega} \times \bar{\Omega}$ such that $\sigma(x, y)=1$ in a neighborhood of the diagonal $\{(x, x) ; x \in \bar{\Omega}\}$ in $\bar{\Omega} \times \bar{\Omega}$. The function $\sigma(x, y)$ depends on the shape of the domain $\Omega$; for example, if $\Omega$ is convex, one may take $\sigma(x, y) \equiv 1$ on $\bar{\Omega} \times \bar{\Omega}$. $\Omega$.

(6) $W 1(x)=c(x)+\int_{\Omega} s(x, y)[1-\sigma(x, y)] d y \leq 0$ in $\Omega$, and $W 1 \not \equiv 0$ in

The operator $W$ is called a second-order Waldenfels operator (cf. [BCP], [Ta3]). The differential operator $P$ is called a diffusion operator which describes analytically a strong Markov process with continuous paths (diffusion process) in the interior $\Omega$. The operator $S_{r}$ is called a second-order Lévy operator which is supposed to correspond to the jump phenomenon in the interior $\Omega$; a Markovian particle moves by jumps to a random point, according to a law determined by the kernel $s(x, y)$ and function $\sigma(x, y)$, in the interior $\Omega$.

Let $L$ be a second-order, boundary condition with real coefficients such that in local coordinates $\left(x_{1}, x_{2}, \ldots, x_{n-1}\right)$ on $\partial \Omega$

$$
\begin{aligned}
L u\left(x^{\prime}\right)= & Q u\left(x^{\prime}\right)+\gamma\left(x^{\prime}\right) u\left(x^{\prime}\right)+\mu\left(x^{\prime}\right) \frac{\partial u}{\partial \nu}\left(x^{\prime}\right)+R_{r} u\left(x^{\prime}\right) \\
:= & \left(\sum_{i, j=1}^{n-1} \alpha^{i j}\left(x^{\prime}\right) \frac{\partial^{2} u}{\partial x_{i} \partial x_{j}}\left(x^{\prime}\right)+\sum_{i=1}^{n-1} \beta^{i}\left(x^{\prime}\right) \frac{\partial u}{\partial x_{i}}\left(x^{\prime}\right)\right)+\gamma\left(x^{\prime}\right) u\left(x^{\prime}\right) \\
& +\mu\left(x^{\prime}\right) \frac{\partial u}{\partial \nu}\left(x^{\prime}\right)+\int_{\Omega} r\left(x^{\prime}, y\right)\left[u(y)-u\left(x^{\prime}\right)\right] d y .
\end{aligned}
$$

Here:

(1) The operator $Q$ is a second-order, degenerate elliptic differential operator with $C^{\infty}$ coefficients on $\partial \Omega$. More precisely, the $\alpha^{i j}$ are the components of a $C^{\infty}$ symmetric contravariant tensor of type $\left(\begin{array}{l}2 \\ 0\end{array}\right)$ on $\partial \Omega$ satisfying

$$
\sum_{i, j=1}^{n-1} \alpha^{i j}\left(x^{\prime}\right) \xi_{i} \xi_{j} \geq 0, \quad x^{\prime} \in \partial \Omega, \quad \xi^{\prime}=\sum_{j=1}^{n-1} \xi_{j} d x_{j} \in T_{x^{\prime}}^{*}(\partial \Omega),
$$

where $T_{x^{\prime}}^{*}(\partial \Omega)$ is the cotangent space of $\partial \Omega$ at $x^{\prime}$.

(2) $L 1=\gamma \in C^{\infty}(\partial \Omega)$ and $\gamma \leq 0$ on $\partial \Omega$.

(3) $\mu \in C^{\infty}(\partial \Omega)$ and $\mu \geq 0$ on $\partial \Omega$.

(4) $\nu=\left(\nu_{1}, \ldots, \nu_{n}\right)$ is the unit interior normal to the boundary $\partial \Omega$.

(5) The integral kernel $r(x, y)$ is the distribution kernel of a properly supported, pseudo-differential operator $R \in L_{1,0}^{0}\left(\mathbf{R}^{n}\right)$ which has the transmission property with respect to the boundary $\partial \Omega$, and $r(x, y) \geq 0$ off the diagonal in $\mathbf{R}^{n} \times \mathbf{R}^{n}$.

The boundary condition $L$ is called a second-order Ventcel' boundary condition (cf. [BCP], [Ta3]). The terms of $L$ are supposed to correspond to the diffusion along the boundary, the absorption phenomenon, the reflection phenomenon, and the inward jump phenomenon from the boundary, respectively. 
In this paper, we prove existence and uniqueness theorems for the following boundary value problem in the framework of Sobolev spaces:

$$
\begin{cases}W u=f & \text { in } \Omega, \\ L u=\varphi & \text { on } \partial \Omega .\end{cases}
$$

To state our fundamental hypotheses concerning the boundary condition $L$, we introduce some notation and definitions.

We let

$$
Y_{i}=\sum_{j=1}^{n-1} \alpha^{i j} \frac{\partial}{\partial x_{j}}, \quad 1 \leq i \leq n-1 ; \quad Y_{0}=\sum_{i=1}^{n-1}\left(\beta_{i}-\sum_{j=1}^{n-1} \frac{\partial \alpha^{i j}}{\partial x_{j}}\right) \frac{\partial}{\partial x_{i}},
$$

and define the Lie algebra $\mathscr{L}(\mathscr{Y})$ over $\mathbf{R}$ generated by the vector fields $Y_{1}, \ldots, Y_{n-1}$ and $Y_{0}$ in the following way: In terms of local coordinates $\left(x_{1}, x_{2}, \ldots, x_{n-1}\right)$ on $\partial \Omega$, we define a mapping

$$
\Psi: \Gamma\left(\partial \Omega, T^{*}(\partial \Omega)\right) \longrightarrow \Gamma(\partial \Omega, T(\partial \Omega))
$$

by the formula

$$
\Psi(\zeta)=\sum_{j=1}^{n-1}\left(\sum_{i=1}^{n-1} \alpha^{i j} \zeta_{i}\right) \frac{\partial}{\partial x_{j}}, \quad \zeta=\sum_{i=1}^{n-1} \zeta_{i} d x_{i}
$$

and

$$
\mathscr{Y}_{1}=\text { the image of } \Psi=\left\{\Psi(\zeta) ; \zeta \in \Gamma\left(\partial \Omega, T^{*}(\partial \Omega)\right)\right\} .
$$

Here $\Gamma\left(\partial \Omega, T^{*}(\partial \Omega)\right)$ and $\Gamma(\partial \Omega, T(\partial \Omega))$ are the space of $C^{\infty}$ covariant vector fields on $\partial \Omega$ and the space of $C^{\infty}$ contravariant vector fields on $\partial \Omega$, respectively. Then we let

$\mathscr{L}(\mathscr{Y})=$ the Lie algebra over $\mathbf{R}$ generated by the vector fields $\mathscr{Y}_{1}$ and $Y_{0}$.

If $x^{\prime}$ is a point of the boundary $\partial \Omega$, then we let

$\omega\left(x^{\prime}\right)=$ the closure of all points $y^{\prime} \in \partial \Omega$ which can be joined to $x^{\prime}$ by a piecewise differentiable curve, of which each differentiable arc is an integral curve of one of the vector fields $\mathscr{Y}_{1}$ and $Y_{0}$.

The set $\omega\left(x^{\prime}\right)$ is called the propagation set of $x^{\prime}$ (cf. [Ta2, Chapter 7]).

Now we can state our fundamental hypotheses for the boundary condition $L$ (cf. Figures 1 and 2): set

(H1) There exists a compact $C^{\infty}$ submanifold $\Sigma$ of codimension one in the

$$
M=\left\{x^{\prime} \in \partial \Omega ; \mu\left(x^{\prime}\right)=0\right\}
$$

such that the Lie algebra $\mathscr{L}(\mathscr{Y})$ has rank $n-1$ at each point of the set $M \backslash \Sigma$.

(H2) There exists a nested family of compact $C^{\infty}$ submanifolds

$$
\begin{aligned}
& \Sigma=\Sigma^{1} \supset \Sigma^{2} \supset \cdots \supset \Sigma^{k} \supset \Sigma^{k+1}=\varnothing, \\
& \operatorname{dim} \Sigma^{j}>\operatorname{dim} \Sigma^{j+1}, \quad j=1, \ldots, k,
\end{aligned}
$$

such that the Lie algebra $\mathscr{L}(\mathscr{Y})$ is transversal to the submanifold $\Sigma^{j}$ at each point $x^{\prime}$ of $\Sigma^{j} \backslash \Sigma^{j+1}, j=1, \ldots, k$, that is, there exists a tangent vector $Y$ in $\mathscr{L}(\mathscr{Y})$ which is transversal to the tangent space $T_{x^{\prime}}\left(\Sigma^{j}\right)$. 


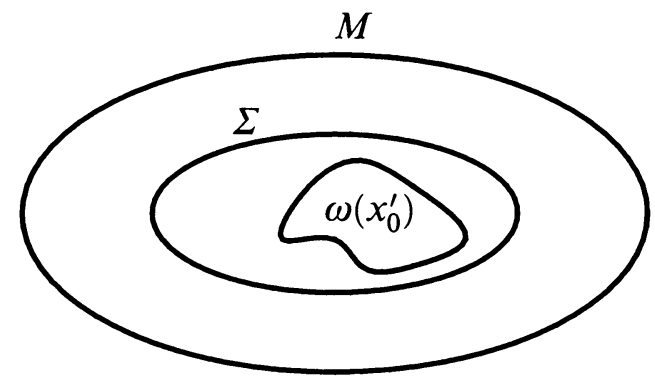

FIGURE 1

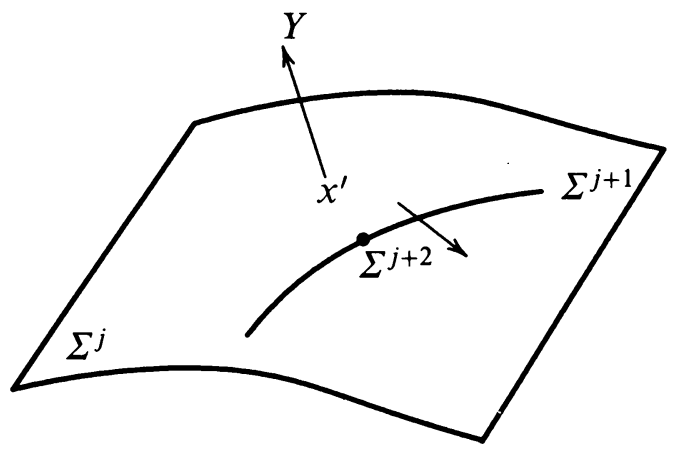

FIGURE 2

(H3) If there exists a point $x_{0}^{\prime}$ of $M$ such that the propagation set $\omega\left(x_{0}^{\prime}\right)$ is entirely contained in $M$, then we have

$$
\left|\gamma\left(x^{\prime}\right)\right|+\int_{\Omega} r\left(x^{\prime}, y\right) d y \not \equiv 0 \text { on } \omega\left(x_{0}^{\prime}\right)
$$

Intuitively, hypotheses (H1) and (H2) imply that a Markovian particle does not stay at any point of the set $M=\left\{x^{\prime} \in \partial \Omega ; \mu\left(x^{\prime}\right)=0\right\}$, where no reflection phenomenon occurs, for any period of time. However, a Markovian particle may be trapped in the set $M$. Hypothesis (H3) is a non-trapping condition for the set $M$; more precisely, hypothesis (H3) implies that, at some point of the set $\omega\left(x_{0}^{\prime}\right)$, a Markovian particle may "disappear" or "move by jumps" to a random point in the interior $\Omega$.

The next theorem asserts that if a Markovian particle moves incessantly both by jumps and continuously in the state space $\bar{\Omega}=\Omega \cup \partial \Omega$, not being trapped in the set $M$ where no reflection phenomenon occurs, then problem $(*)$ is uniquely solvable in the framework of Sobolev spaces:

Theorem 1. Assume that hypotheses $(\mathrm{H} 1),(\mathrm{H} 2)$ and $(\mathrm{H} 3)$ are satisfied. Then, for any $f \in H^{s}(\Omega)$ and any $\varphi \in H^{s-1 / 2}(\partial \Omega)$ with $s>1 / 2$, there exists $a$ unique solution $u \in H^{s}(\Omega)$ of the problem

$$
\begin{cases}W u=f & \text { in } \Omega \\ L u=\varphi & \text { on } \partial \Omega\end{cases}
$$


Furthermore, we consider the case when $\alpha^{i j} \equiv 0$ on $\partial \Omega$ :

$$
\begin{aligned}
L_{o} u\left(x^{\prime}\right)= & \sum_{i=1}^{n-1} \beta^{i}\left(x^{\prime}\right) \frac{\partial u}{\partial x_{i}}\left(x^{\prime}\right)+\gamma\left(x^{\prime}\right) u\left(x^{\prime}\right)+\mu\left(x^{\prime}\right) \frac{\partial u}{\partial \nu}\left(x^{\prime}\right) \\
& +\int_{\Omega} r\left(x^{\prime}, y\right)\left[u(y)-u\left(x^{\prime}\right)\right] d y .
\end{aligned}
$$

The fundamental hypothesis for the boundary condition $L_{o}$ is stated as follows:

(H4) The vector field $\beta=\sum_{i} \beta^{i} \partial / \partial x_{i}$ is non-zero on $M=\left\{x^{\prime} \in \partial \Omega\right.$; $\left.\mu\left(x^{\prime}\right)=0\right\}$, and any maximal integral curve of $\beta$ is not entirely contained in $M$.

The vector field $\beta$ coincides with the drift vector field $Y_{0}$, since $\alpha^{i j} \equiv 0$ on $\partial \Omega$. Hence the intuitive meaning of hypothesis (H4) is that a Markovian particle "goes through" the set $M$ where no reflection phenomenon occurs; that is, hypothesis $(\mathrm{H} 4)$ is a non-trapping condition for the set $M$, similar to hypothesis (H3) in Theorem 1.

Then we can prove the following result:

Theorem 2. Let $S \in L_{1,0}^{1-\kappa}\left(\mathbf{R}^{n}\right)$ with $\kappa>0$ and $R \in L_{1,0}^{-\theta}\left(\mathbf{R}^{n}\right)$ with $\theta>0$. Assume that hypothesis ( $\mathrm{H} 4)$ is satisfied. Then, for any $f \in H^{s}(\Omega)$ and any $\varphi \in H^{s+1 / 2}(\partial \Omega)$ with $s>-1 / 2$, there exists a unique solution $u \in H^{s+1}(\Omega)$ of the problem

$$
\begin{cases}W u=f & \text { in } \Omega \\ L_{o} u=\varphi & \text { on } \partial \Omega\end{cases}
$$

Theorems 1 and 2 extend and improve substantially Theorem 3 of Paneyakh [Pa] and Théorème 2 of Taira [Ta1], which only treated the case when $S \equiv 0$ and $R \equiv 0$.

\section{Proof of Theorem 1}

(1) First, we show that the boundary value problem

$$
\begin{cases}W u=f & \text { in } \Omega, \\ L u=\varphi & \text { on } \partial \Omega\end{cases}
$$

can be reduced to the study of an operator on the boundary.

Now we consider the following Dirichlet problem:

$$
\begin{cases}W v=f & \text { in } \Omega \\ v=0 & \text { on } \partial \Omega\end{cases}
$$

The existence and uniqueness theorem for problem (D) is well established in the framework of Sobolev spaces (cf. [Ho, Chapter XX], [RS, Chapter 3]). Indeed, it suffices to note that the operator $W$ is a perturbation of a compact operator, in the framework of Sobolev spaces, to the second-order elliptic differential operator $P$, since $W=P+S_{r}$ and $S_{r}$ is of order $2-\kappa<2$. We let

$$
v=\mathscr{G} f \text {. }
$$

Then it follows that a function $u$ is a solution of problem $(*)$ if and only if the function $w=u-v$ is a solution of the problem:

$$
\begin{cases}W w=0 & \text { in } \Omega, \\ L w=\varphi-L v=\varphi-L \mathscr{G} f & \text { on } \partial \Omega\end{cases}
$$


But we know that every solution $w$ of the homogeneous equation $W w=0$ in $\Omega$ can be expressed as follows:

$$
w=\mathscr{P} \psi .
$$

Thus, by using the operators $\mathscr{G}$ and $\mathscr{P}$, one can reduce the study of problem $(*)$ to that of the equation

$$
\text { (+) } \quad T \psi:=L \mathscr{P} \psi=\varphi-L \mathscr{G} f \text { on } \partial \Omega \text {. }
$$

More precisely, we can formulate this reduction to the boundary in the framework of Sobolev spaces as follows:

Proposition 2.1. For functions $f \in H^{s}(\Omega)$ and $\varphi \in H^{s-1 / 2}(\partial \Omega)$ with $s>1 / 2$, there exists a solution $u \in H^{s}(\Omega)$ of problem $(*)$ if and only if there exists a solution $\psi \in H^{s-1 / 2}(\partial \Omega)$ of equation $(+)$. Furthermore, the solutions $u$ and $\psi$ are related as follows: $u=\mathscr{G} f+\mathscr{P} \psi$ where $\mathscr{G} f \in H^{s+2}(\Omega)$ and $\mathscr{P} \psi \in H^{s}(\Omega)$.

(2) Next, we show that the operator

$$
\begin{aligned}
T \psi\left(x^{\prime}\right)= & \sum_{i, j=1}^{n-1} \alpha^{i j}\left(x^{\prime}\right) \frac{\partial^{2} \psi}{\partial x_{i} \partial x_{j}}\left(x^{\prime}\right)+\sum_{i=1}^{n-1} \beta^{i}\left(x^{\prime}\right) \frac{\partial \psi}{\partial x_{i}}\left(x^{\prime}\right)+\gamma\left(x^{\prime}\right) \psi\left(x^{\prime}\right) \\
& +\mu\left(x^{\prime}\right) \frac{\partial}{\partial \nu}(\mathscr{P} \psi)\left(x^{\prime}\right)+\int_{\Omega} r\left(x^{\prime}, y\right)\left[\mathscr{P} \psi(y)-\psi\left(x^{\prime}\right)\right] d y
\end{aligned}
$$

is a second-order, pseudo-differential operator on the boundary $\partial \Omega$.

Indeed, we have the following:

(a) It is known (cf. [Ho, Chapter XX], [RS, Chapter 3]) that the operator

$$
\Pi: \psi\left(x^{\prime}\right) \longmapsto \frac{\partial}{\partial \nu}(\mathscr{P} \psi)\left(x^{\prime}\right)
$$

is a first-order, elliptic pseudo-differential operator on $\partial \Omega$.

(b) Since the pseudo-differential operator $R \in L_{1,0}^{0}\left(\mathbf{R}^{n}\right)$ has the transmission property with respect to $\partial \Omega$, it follows (cf. [Bo], [RS, Chapter 2]) that the operator

$$
U: \psi\left(x^{\prime}\right) \longmapsto \int_{\Omega} r\left(x^{\prime}, y\right) \mathscr{P} \psi(y) d y
$$

is a pseudo-differential operator of order zero on $\partial \Omega$.

(3) We study the operator $T$ in question and show that conditions (H1) and (H2) are sufficient for the validity of a priori estimates for $T$.

First we prove an a priori estimate for $T$ outside the submanifold $\Sigma$ :

Lemma 2.2. Assume that hypothesis $(\mathrm{H} 1)$ is satisfied. Let $\sigma \in \mathbf{R}$, and let $\chi$ be a function in $C_{0}^{\infty}(\partial \Omega \backslash \Sigma)$. Then, for each $\varepsilon>0$, there exists a constant $C_{\varepsilon, \chi}>0$ such that we have for all $\psi \in C^{\infty}(\partial \Omega)$

$$
|\chi \psi|_{H^{o}(\partial \Omega)} \leq \varepsilon\left(|T \psi|_{H^{\sigma}(\partial \Omega)}+|\psi|_{H^{\sigma}(\partial \Omega)}\right)+C_{\varepsilon, \chi}|\psi|_{H^{o-1}(\partial \Omega)} .
$$

Proof. We remark that the pseudo-differential operator $T$ is of the form:

$$
\begin{aligned}
T= & \sum_{i, j=1}^{n-1} \alpha^{i j} \frac{\partial^{2}}{\partial x_{i} \partial x_{j}}+\sum_{i=1}^{n-1} \beta^{i} \frac{\partial}{\partial x_{i}}+\mu \Pi+\left(\gamma-\int_{\Omega} r(\cdot, y) d y\right) \\
& + \text { a pseudo-differential operator } U \text { of order zero. }
\end{aligned}
$$


That is, the pseudo-differential operator $T$ is a perturbation of a bounded operator, in the framework of Sobolev spaces, to the differential operator

$$
\sum_{i, j} \alpha^{i j} \partial^{2} / \partial x_{i} \partial x_{j}+\sum_{i} \beta^{i} \partial / \partial x_{i}+\mu \Pi+\gamma
$$

which is studied in detail in Paneyakh [Pa]. Therefore, the desired estimate (2.1) can be obtained as in [Pa, Theorem 2.5].

Similarly, we can prove an a priori estimate for $T$ in a tubular neighborhood of the submanifold $\Sigma$, just as in [Pa, Theorem 3.1]:

Lemma 2.3. Assume that hypothesis ( $\mathrm{H} 2)$ is satisfied. Let $\sigma \in \mathbf{R}$, and let $\Xi$ be a sufficiently small tubular neighborhood of the submanifold $\Sigma$. Then, for each $\varepsilon>0$, there exists a constant $C_{\varepsilon}>0$ such that we have for all $\psi \in C^{\infty}(\partial \Omega)$ with $\operatorname{supp} \psi \subset \Xi$

$$
|\psi|_{H^{\sigma}(\partial \Omega)} \leq \varepsilon|T \psi|_{H^{\sigma}(\partial \Omega)}+C_{\varepsilon}|\psi|_{H^{\sigma-1}(\partial \Omega)} .
$$

Therefore, combining estimates (2.1) and (2.2), together with a partition of unity, we have the following fundamental a priori estimate for the operator $T$ (cf. [Pa, Theorem 4.1]):

Proposition 2.4. Assume that hypotheses $(\mathrm{H} 1)$ and $(\mathrm{H} 2)$ are satisfied, and let $\sigma \in \mathbf{R}$. Then, for each $\varepsilon>0$, there exists a constant $C_{\varepsilon}>0$ such that we have for all $\psi \in C^{\infty}(\partial \Omega)$

$$
|\psi|_{H^{\sigma}(\partial \Omega)} \leq \varepsilon|T \psi|_{H^{o}(\partial \Omega)}+C_{\varepsilon}|\psi|_{H^{\sigma-1}(\partial \Omega)} .
$$

(4) We associate with equation $(+)$ a densely defined, closed linear operator

$$
\mathscr{T}: H^{s-1 / 2}(\partial \Omega) \longrightarrow H^{s-1 / 2}(\partial \Omega)
$$

as follows:

$(\alpha)$ The domain $D(\mathscr{T})$ of $\mathscr{T}$ is the space

$$
D(\mathscr{T})=\left\{\psi \in H^{s-1 / 2}(\partial \Omega) ; T \psi \in H^{s-1 / 2}(\partial \Omega)\right\} .
$$

(ß) $\mathscr{T} \psi=T \psi, \psi \in D(\mathscr{T})$.

Then, by using Proposition 2.4 as in [Pa, Theorem 4.2], we can obtain the following:

Theorem 2.5. Assume that hypotheses $(\mathrm{H1})$ and $(\mathrm{H} 2)$ are satisfied, and let $s \in$ R. Then we have:

(i) The operator $\mathscr{T}: H^{s-1 / 2}(\partial \Omega) \longrightarrow H^{s-1 / 2}(\partial \Omega)$ is a Fredholm operator with index zero, that is,

$$
\text { ind } \mathscr{T}:=\operatorname{dim} N(\mathscr{T})-\operatorname{codim} R(\mathscr{T})=0 \text {. }
$$

(ii) The null space $N(\mathscr{T})$ consists of $C^{\infty}$ functions on $\partial \Omega$.

(5) Now we prove existence and uniqueness result for problem $(*)$ :

Problem $(*)$ has a unique solution $u \in H^{s}(\Omega)$ for any

$f \in H^{s}(\Omega)$ and any $\varphi \in H^{s-1 / 2}(\partial \Omega)$ with $s>1 / 2$. 
(5-1) To do so, we first prove uniqueness result for problem $(*)$ :

$$
\left\{\begin{array}{l}
u \in H^{s}(\Omega), W u=0 \text { in } \Omega, L u=0 \text { on } \partial \Omega \\
\Longrightarrow u=0 \text { in } \Omega .
\end{array}\right.
$$

In view of Proposition 2.1, part (ii) of Theorem 2.5 implies that

$$
\left\{\begin{array}{l}
u \in H^{s}(\Omega), W u=0 \text { in } \Omega, L u=0 \text { on } \partial \Omega \\
\Longrightarrow u \in C^{\infty}(\bar{\Omega}) .
\end{array}\right.
$$

Therefore, uniqueness result (2.4) is an immediate consequence of the following maximum principle:

Proposition 2.6. Assume that hypothesis (H3) is satisfied. Then we have:

$$
\left\{\begin{array}{l}
u \in C^{2}(\bar{\Omega}), W u \geq 0 \text { in } \Omega, L u \geq 0 \text { on } \partial \Omega \\
\Longrightarrow u \leq 0 \text { on } \bar{\Omega} .
\end{array}\right.
$$

Proof. We only have to consider the case when $u$ is not a constant. Assume to the contrary that

$$
m=\max _{\bar{\Omega}} u>0
$$

Then, applying the strong maximum principle (cf. [BCP, Théorème VII]) to the operator $W$, we obtain that there exists a point $x_{0}^{\prime}$ of $\partial \Omega$ such that

$$
\left\{\begin{array}{l}
u\left(x_{0}^{\prime}\right)=m \\
u(x)<u\left(x_{0}^{\prime}\right) \text { for all } x \in \Omega
\end{array}\right.
$$

Furthermore, it follows from an application of the boundary point lemma (cf. [BCP, Théorème VIII]) that

$$
\frac{\partial u}{\partial \nu}\left(x_{0}^{\prime}\right)<0
$$

This implies that $\mu\left(x_{0}^{\prime}\right)=0$, that is, $x_{0}^{\prime} \in M$, since we have

$$
0 \leq L u\left(x_{0}^{\prime}\right) \leq \mu\left(x_{0}^{\prime}\right) \frac{\partial u}{\partial \nu}\left(x_{0}^{\prime}\right) .
$$

But, applying the sharp maximum principle (cf. [Ta2, Theorem 7.2.1]) to our situation, we find that:

$u \equiv m$ throughout the propagation set $\omega\left(x_{0}^{\prime}\right)$ of $x_{0}^{\prime} \in M$.

Therefore, by virtue of hypothesis (H3), we can find a point $y_{0}^{\prime}$ of $\partial \Omega$ such that $u\left(y_{0}^{\prime}\right)=m$, and that (cf. Figure 1):

(a) If $\omega\left(x_{0}^{\prime}\right) \not \subset M$, then we have $y_{0}^{\prime} \notin M$ and so

$$
\mu\left(y_{0}^{\prime}\right)>0, \quad \frac{\partial u}{\partial \nu}\left(y_{0}^{\prime}\right)<0 .
$$

(b) If $\omega\left(x_{0}^{\prime}\right) \subset M$, then we have $y_{0}^{\prime} \in \omega\left(x_{0}^{\prime}\right)$ and

$$
\gamma\left(y_{0}^{\prime}\right) u\left(y_{0}^{\prime}\right)+\int_{\Omega} r\left(y_{0}^{\prime}, y\right)\left[u(y)-u\left(y_{0}^{\prime}\right)\right] d y<0 .
$$

This contradicts the hypothesis: $L u \geq 0$ on $\partial \Omega$. Indeed, we then have

$$
L u\left(y_{0}^{\prime}\right) \leq \mu\left(y_{0}^{\prime}\right) \frac{\partial u}{\partial \nu}\left(y_{0}^{\prime}\right)+\gamma\left(y_{0}^{\prime}\right) u\left(y_{0}^{\prime}\right)+\int_{\Omega} r\left(y_{0}^{\prime}, y\right)\left[u(y)-u\left(y_{0}^{\prime}\right)\right] d y<0
$$


(5-2) In view of Proposition 2.1, uniqueness result (2.4) implies that the operator $\mathscr{T}: H^{s-1 / 2}(\partial \Omega) \longrightarrow H^{s-1 / 2}(\partial \Omega)$ is injective, that is, $\operatorname{dim} N(\mathscr{T})=0$. But, part (i) of Theorem 2.5 tells us that $\mathscr{T}$ is a Fredholm operator with index zero. Thus we find that $\operatorname{codim} R(\mathscr{T})=\operatorname{dim} N(\mathscr{T})=0$, which proves that the operator $\mathscr{T}$ is surjective; hence it is bijective. Therefore, existence and uniqueness result (2.3) follows by again using Proposition 2.1.

The proof of Theorem 1 is now complete.

\section{Proof of Theorem 2}

The idea of the proof of Theorem 2 is essentially the same as that of Theorem 1.

(1) First, arguing as in step (1) of the proof of Theorem 1, we are reduced to the study of the pseudo-differential equation:

$$
(++) \quad T_{o} \psi:=L_{o} \mathscr{P} \psi=\varphi-L_{o} \mathscr{G} f \text { on } \partial \Omega \text {. }
$$

But the operator $T_{o}$ is of the form:

$$
\begin{aligned}
T_{o}= & \sum_{i=1}^{n-1} \beta^{i} \frac{\partial}{\partial x_{i}}+\mu \Pi+\left(\gamma-\int_{\Omega} r(\cdot, y) d y\right) \\
& + \text { a pseudo-differential operator } U \text { of order }-\theta .
\end{aligned}
$$

Therefore, just as in [Ta1, Lemme 3.6], we can prove the following:

Proposition 3.1. If hypothesis ( $\mathrm{H} 4)$ is satisfied, then there exists a parametrix $E_{o}$ for $T_{o}$ which maps $H^{\sigma}(\partial \Omega)$ continuously into itself for all $\sigma \in \mathbf{R}$.

(2) We associate with equation $(++)$ a densely defined, closed linear operator

$$
\mathscr{T}_{o}: H^{s+1 / 2}(\partial \Omega) \longrightarrow H^{s+1 / 2}(\partial \Omega)
$$

as follows:

$(\alpha)$ The domain $D\left(\mathscr{T}_{o}\right)$ of $\mathscr{T}_{0}$ is the space

$$
D\left(\mathscr{T}_{o}\right)=\left\{\psi \in H^{s+1 / 2}(\partial \Omega) ; T_{o} \psi \in H^{s+1 / 2}(\partial \Omega)\right\} .
$$

(ß) $\mathscr{T}_{o} \psi=T_{o} \psi, \psi \in D\left(\mathscr{T}_{o}\right)$. 2.5:

Then we have the following result for the operator $\mathscr{T}_{o}$, analogous to Theorem

Theorem 3.2. Assume that hypotheses $(\mathrm{H} 4)$ is satisfied. Then we have:

(i) The operator $\mathscr{T}_{0}: H^{s+1 / 2}(\partial \Omega) \longrightarrow H^{s+1 / 2}(\partial \Omega)$ is a Fredholm operator with index zero.

(ii) The null space $N\left(\mathscr{T}_{0}\right)$ consists of $C^{\infty}$ functions on $\partial \Omega$.

Proof. To prove assertion (i), we remark that the operator

$$
\left(W, L_{o}\right)=\left(P+S_{r}, L_{0}+U\right): H^{s+1}(\Omega) \longrightarrow H^{s}(\Omega) \times H^{s+1 / 2}(\partial \Omega)
$$

is a perturbation of a compact operator to the operator $\left(P, L_{0}\right)$, since $S_{r}$ is of order $1-\kappa<1$ and $U$ is of order $-\theta<0$. Here $L_{0}=\mu \partial / \partial \nu+\sum_{i} \beta^{i} \partial / \partial x_{i}+$ $\gamma-\int_{\Omega} r(\cdot, y) d y$. But, by [Ta 1, Corollaire 4.3], we know that if hypothesis (H4) 
is satisfied, then we have ind $\left(P, L_{0}\right)=0$. Therefore, we obtain that

$$
\text { ind } \mathscr{T}_{o}=\operatorname{ind}\left(W, L_{o}\right)=\operatorname{ind}\left(P, L_{0}\right)=0 \text {. }
$$

Assertion (ii) is an immediate consequence of Proposition 3.1.

By virtue of Theorem 3.2, Theorem 2 can be proved just as in the proof of Theorem 1 .

\section{REFERENCES}

[BCP] J.-M. Bony, P. Courrège, and P. Priouret, Semi-groupes de Feller sur une variété à bord compacte et problèmes aux limites intégro-différentiels du second ordre donnant lieu au principe du maximum, Ann. Inst. Fourier (Grenoble) 18 (1968), 369-521.

[Bo] L. Boutet de Monvel, Boundary problems for pseudo-differential operators, Acta Math. 126 (1971), 11-51.

[Ho] L. Hörmander, The analysis of linear partial differential operators III, Springer-Verlag, Berlin, Heidelberg, New York, and Tokyo, 1985.

[Pa] B. P. Paneyakh, Some boundary value problems for elliptic equations, and the Lie algebras associated with them, Mat. Sb. 126 (1985), 215-246; English transl., Math. USSR-Sb. 54 (1986), 207-237.

[RS] S. Rempel and B.-W. Schulze, Index theory of elliptic boundary problems, Akademie-Verlag, Berlin, 1982.

[Ta1] K. Taira, Sur le problème de la dérivée oblique I, J. Math. Pures Appl. 57 (1978), 379-395.

[Ta2] _ Diffusion processes and partial differential equations, Academic Press, San Diego, New York, London, and Tokyo, 1988.

[Ta3] __ On the existence of Feller semigroups with boundary conditions, Mem. Amer. Math. Soc., no. 475, Amer. Math. Soc., Providence, RI, 1992.

INSTitute of Mathematics, UNiversity OF TSUKuba, Tsukuba 305, JAPAN

E-mail address: taira@math.tsukuba.ac.jp 